\title{
Commentary Endocannabinoid Regulation of Cocaine Reinforcement: an Upper or Downer?
}

\author{
Dan P Covey', Natalie E Zlebnik' and Joseph F Cheer*,1,2 \\ 'Department of Anatomy and Neurobiology, University of Maryland School of Medicine, Baltimore, MD, USA; '2Department of Psychiatry, \\ University of Maryland School of Medicine, Baltimore, MD, USA
}

Neuropsychopharmacology (2016) 4I, 2189-2191; doi:10.1038/npp.2016.25

Mounting clinical and preclinical work implicates the endocannabinoid $(\mathrm{eCB})$ system as a prominent regulator of drug reinforcement (Parsons and Hurd, 2015). Specifically, the cannabinoid type 1 (CB1) receptor has emerged as a major conduit by which addictive drugs access brain reward circuits to exploit reinforcement mechanisms and motivate drug abuse processes. Pharmacologically or genetically increasing or decreasing CB1 receptor activity, respectively, increases or decreases drug effects on neural activity related to reward pursuit and drug seeking behavior. Accordingly, pharmacotherapies targeting CB1 receptors have garnered much interest and have demonstrated efficacy for suppressing drug abuse and addiction, but their utility has been mired due to non-specific side effects, such as depression and anxiety (Lazary et al, 2011). Thus, future treatment development requires a more detailed, mechanistic understanding of the precise role CB1 receptors serve in specific neural circuits and behaviors.

This endeavor is somewhat hindered by the complexity of the eCB system. CB1 receptors are ubiquitously expressed throughout the brain in neurons, glia, and different cell compartments such as endosomes and mitochondria. Moreover, $\mathrm{CB} 1$ receptor signaling occurs in a unique manner. The primary endogenous ligands for the $\mathrm{CB} 1$ receptor, anandamide and 2-arachidonoylglycerol (2-AG), are released from cell bodies in a retrograde fashion (Figure 1). These lipophilic eCBs are also not pre-packaged in vesicular stores but are enzymatically synthesized 'on-demand' via cleavage of membrane phospholipids. Thus, eCB signaling is triggered by the activation of the requisite biosynthetic machinery located at a site postsynaptic to CB1 receptor-expressing terminals. Due to the inhibitory nature of the $G_{i / o}$-coupled $\mathrm{CB} 1$ receptors, this arrangement permits a negative feedback mechanism that allows numerous cell types to bidirectionally autoregulate activity by suppressing excitatory (eg, glutamate) or inhibitory (eg, GABA) transmission, resulting in inhibition or disinhibition, respectively, of the postsynaptic

*Correspondence: Dr J Cheer, Department of Anatomy and Neurobiology, University of Maryland School of Medicine, 20 Penn Street, Baltimore, MD 21201, USA, Tel: +I 410 7060lI2, E-mail: jchee00।@umaryland.edu cell. For example, dopamine function within the mesocorticolimbic circuit, which is central to drug reinforcement, may be potentiated or inhibited depending on which afferent inputs are modulated by $\mathrm{CB} 1$ receptor binding. Unfortunately, current understanding of how $\mathrm{CB} 1$ receptors regulate drug reinforcement mechanisms in situ is largely based on work using pharmacological manipulations, which alter CB1 receptors globally and are therefore not suitable for distinguishing behaviors driven by CB1 receptors on separate cell types.

In this issue, Martín-García et al used transgenic mice to resolve how $\mathrm{CB} 1$ receptors located on glutamatergic vs GABAergic neurons differentially contribute to cocaine reinforcement. Cre/lox recombination technology was employed to generate mice lacking CB1 receptors on either glutamatergic or GABAergic cell populations. Mice bearing a CB1 receptor gene $\left(C N R 1^{\text {loxP/loxP }}\right)$ flanked by two loxP sites were crossed with mice expressing Cre under the regulatory control of the NEX or Dlx5/6 locus. Because Cre excises DNA segments flanked by loxP sites, transgenic offspring lacked $\mathrm{CB} 1$ receptors on either cortical glutamate neurons $\left(C N R 1^{\text {loxP/loxP }} / \mathrm{Nex}-\mathrm{Cr} e^{+/-}\right.$; referred to as Glu-CB1-KOs) or GABAergic forebrain (CNR1 loxP/loxP $/ D l \times 5 / 6-C r e^{+/-}$; referred to as GABA-CB1-KOs) neurons. $\mathrm{CB} 1$ receptor expression in GABA-CB1-KOs was strikingly similar to complete CB1KOs, particularly when compared with Glu-CB1-KOs. This is consistent with the greater overall $\mathrm{CB} 1$ receptor expression on GABA vs glutamate neurons reported in prior work using these transgenic lines, which has found marked differences between Glu-CB1-KOs and GABA-CB1-KOs in measures of feeding, anxiety, and fear (Lutz et al, 2015; Busquets-Garcia et al, 2015). Thus, CB1 receptors on excitatory vs inhibitory terminals may differentially control motivated behaviors.

To assess the cell type-specific contribution of CB1 receptors to cocaine reinforcement, Martín-García et al evaluated these transgenic mice on measures of cocaine self-administration. Results suggest that GABA-CB1-KO mice are more sensitive to cocaine's reinforcing effects, as indicated by a leftward shift in the dose-response curve under a fixed ratio-2 reinforcement schedule and an upward shift in their dose-response curve under a progressive ratio reinforcement schedule. These findings indicate that CB1 


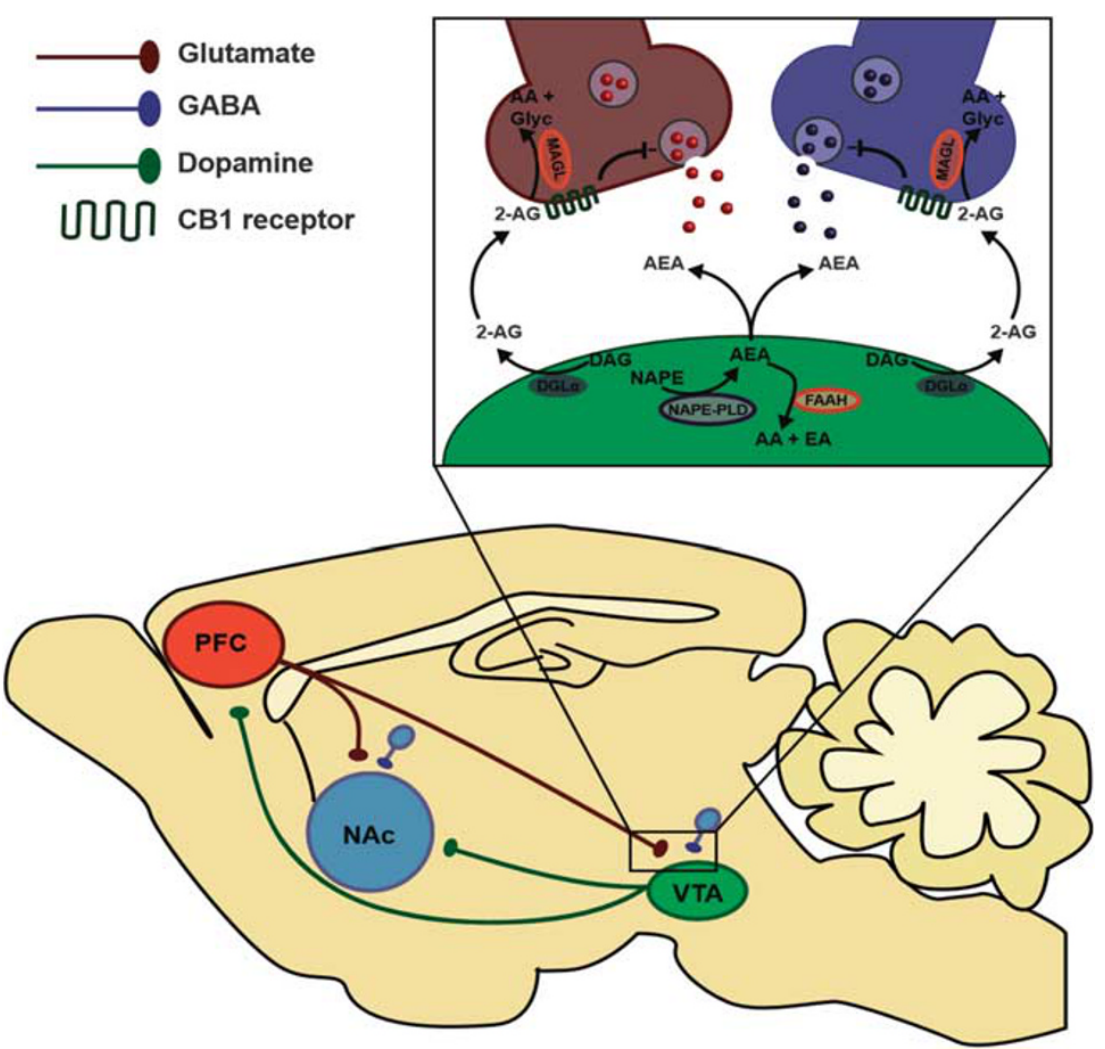

Figure I Endocannabinoid regulation of mesocorticolimbic dopamine circuit. Dopamine neurons in the ventral tegmental area (VTA) project to the nucleus accumbens (NAc) and prefrontal cortex (PFC). Glutamatergic projections from the PFC and GABAergic interneurons both synapse onto NAc and VTA neurons, and express presynaptic CBI receptors (inset). Biosynthesis of 2-arachidonoylglycerol (2-AG) and andandamide (AEA) occurs postsynaptically. 2-AG synthesis occurs via hydrolysis of 1,2-diacylglycerol (DAG) by DAG lipase (DAGL $\alpha$ ). AEA is synthesized from phospholipid precursors (eg, $\mathrm{N}$-arachidonoyl phosphatidylethanolamine; NAPE) via a phospholipase D (NAPE-PLD). Synthetic machinery opposes CBI receptor-expressing terminals, which are suppressed by 2-AG and AEA. Degradative enzymes terminate signaling. Monoacylglycerol lipase (MAGL), located presynaptically, hydrolyzes 2-AG to arachidonic acid (AA) and glycerol (Glyc). Fatty acid amide hydrolase, located postsynaptically, hydrolyzes AEA to AA and ethanolamine (EA). For simplicity, only projections onto the VTA are shown, but similar structure and function is present throughout the brain. Projections also important to the regulation of this circuit and modified by eCBs are reviewed in detail by Parsons and Hurd, 2015, and include dopaminergic projections to the hippocampus and amygdala; GABAergic projections from the NAc back to the VTA and onto output nuclei of the basal ganglia; and glutamatergic projections from the hippocampus and amygdala to the NAc.

receptors on GABA neurons normally curtail cocaine's primary reinforcing properties, and a presumed increase in GABAergic transmission following $\mathrm{CB} 1$ receptor deletion may potentiate cocaine reinforcement. Alternatively, Glu-CB1-KOs exhibited an increase in cue-induced reinstatement of cocaine seeking, while GABA-CB1-KOs did not differ from WT controls. Thus, the primary and secondary reinforcing effects of cocaine may be differentially regulated by $\mathrm{CB} 1$ receptors on glutamatergic vs GABAergic neurons.

Martín-García et al also measured cocaine-evoked dopamine release in the nucleus accumbens (NAc) using microdialysis. Drug-evoked increases in accumbal dopamine arise from drug-induced alterations in mesocorticolimbic function and mediate the primary reinforcing and rewarding effects of abused drugs, including cocaine (Nestler, 2005). Moreover, cocaine-evoked increases in NAc dopamine release require VTA CB1 receptor signaling (Cheer et al, 2007). Recent work demonstrates that cocaine facilitates NAc dopamine by mobilizing 2-AG in the VTA onto CB1 receptor-expressing GABAergic terminals to disinhibit VTA dopamine neurons (Wang et al, 2015). Paradoxically, Martín-García et al found that cocaine elevated dopamine to a much greater degree in GABA-CB1-KOs. Lacking the inhibitory influence of presynaptic $\mathrm{CB} 1$ receptors, GABACB1-KOs should exhibit potentiated GABAergic transmission, which, according to the above model, should suppress cocaine-evoked NAc dopamine release. However, additional neural structures clearly regulate cocaine effects on the brain and widespread alterations in circuit function may be expected following the profound loss of $\mathrm{CB} 1$ receptors in GABA-CB1-KOs, making it difficult to identify the specific factors driving this effect.

This study takes an important leap forward in understanding $\mathrm{CB} 1$ receptor function, as prior genetic assessments have used full KOs, bearing global deletion of the CB1 receptor. While the targeted genetic $\mathrm{KO}$ model utilized by Martín-García et al offers a more refined approach, broad changes in gene function that are present throughout the life-span nonetheless remain an important confound. Developmental changes and compensatory adaptations, in particular, are a major concern when assessing the phenotypic consequences resulting from lack of normal gene expression. Alternative approaches that have been rapidly advancing allow conditional and inducible manipulations 
that permit spatial and temporal control of gene function, offering a finer tool for building upon this work and further dissecting how distinct $\mathrm{CB} 1$ receptor populations regulate specific behaviors.

One potential approach is, rather than to use Cre mouse lines, to deliver Cre recombinase within targeted brain regions of the adult animal using viral vector infusions. Cell-type specificity is accomplished by packaging Cre into vectors (eg, adeno-associated virus (AAV) or lentivirus) under the regulatory control of a known promoter sequence. Infusing promoter-driven, Cre-expressing viruses into the brain of floxed mice produces genetic deletions restricted to the area of viral spread and confined to a specific cell type. This strategy also allows the introduction of certain proteins (eg, opsins or DREADDs) to offer optogenetic or chemogenetic control of Cre-expressing cells. Employing this approach in the $\mathrm{CB1}^{\text {flflf }}$ mice used by Martín-García et al would permit a more selective assessment of how CB1 receptors on GABA or glutamate neurons within targeted circuits contribute to behavior. However, the relatively small packaging capacity of AAVs limits its use for delivering larger genes. Alternative approaches include the use of short-hairpin RNA to elicit site- and cell-type specific gene knockdown, tetracycline-regulated promoter (TetOp) systems to temporally control gene expression, or CRISPR for targeted genome editing across species and cell types. These approaches may also be used to manipulate CB1 receptor function indirectly by targeting the $\mathrm{eCB}$ synthetic or degradative enzymes, which could offer important insight for eCB-based therapeutics. Because eCBs are produced 'on-demand' in a synapse-specific manner, altering the enzymatic regulation of eCBs may preferentially modify circuits that are engaged by distinct stimuli (eg, drug cues) and avoid adverse side effects caused by global CB1 receptor manipulations.

The study by Martín-García et al highlights a complex feature of the eCB system, identifying divergent contributions of cell type-specific populations of $\mathrm{CB} 1$ receptors in the neurobehavioral effects of cocaine. Recent advances in molecular techniques that allow specific neural circuits to be spatially and temporally resolved in awake, behaving animals have transformed the neuroscientist's toolbox and will be important for moving this work forward. These novel approaches offer an unprecedented opportunity to unravel the complexity of this important neuromodulatory network and may pave the way for novel eCB-based treatments for drug abuse and addiction.

\section{FUNDING AND DISCLOSURE}

The authors declare no conflict of interest.

\section{REFERENCES}

Busquets-Garcia A, Desprez T, Metna-Laurent M, Bellocchio L, Marsicano G, Soria-Gomez E (2015). Dissecting the cannabinergic control of behavior: the where matters. Bioessays 37: 1215-1225.

Cheer JF, Wassum KM, Sombers LA, Heien ML, Ariansen JL, Aragona BJ et al (2007). Phasic dopamine release evoked by abused substances requires cannabinoid receptor activation. J Neurosci 27: 791-795.

Lazary J, Juhasz G, Hunyady L, Bagdy G (2011). Personalized medicine can pave the way for the safe use of $\mathrm{CB}(1)$ receptor antagonists. Trends Pharmacol Sci 32: 270-280.

Lutz B, Marsicano G, Maldonado R, Hillard CJ (2015). The endocannabinoid system in guarding against fear, anxiety and stress. Nat Rev Neurosci 16: 705-718.

Martín-García E, Bourgoin L, Cathala A, Kasanetz F, Mondesir M, Gutiérrez-Rodriguez A et al (2015). Differential control of cocaine self-administration by GABAergic and glutamatergic CB1 cannabinoid receptors. Neuropsychopharmacology (in press).

Nestler EJ (2005). Is there a common molecular pathway for addiction? Nat Neurosci 8: 1445-1449.

Parsons LH, Hurd YL (2015). Endocannabinoid signalling in reward and addiction. Nat Rev Neurosci 16: 579-594.

Wang H, Treadway T, Covey DP, Cheer JF, Lupica CR (2015). Cocaine induced endocannabinoid mobilization in the ventral tegmental area. Cell Rep 12: 1997-2008. 